\title{
Multi-Trait Analysis of GWAS and Biological Insights Into Cognition: A Response to Hill (2018)
}

\author{
Max Lam, ${ }^{1}$ Joey W. Trampush, ${ }^{2}$ Jin Yu, ${ }^{3}$ Emma Knowles, ${ }^{4}$ Srdjan Djurovic, ${ }^{5,6}$ Ingrid Melle, ${ }^{6,7}$ \\ Kjetil Sundet, ${ }^{7,8}$ Andrea Christoforou, ${ }^{9}$ Ivar Reinvang, ${ }^{8}$ Pamela DeRosse, ${ }^{3}$ Astri J. Lundervold, ${ }^{10}$ \\ Vidar M. Steen, ${ }^{6,9}$ Thomas Espeseth, ${ }^{7,8}$ Katri Räikkönen, ${ }^{11}$ Elisabeth Widen, ${ }^{12}$ Aarno Palotie, ${ }^{12,13,14}$ \\ Johan G. Eriksson, ${ }^{15,16,17}$ Ina Giegling, ${ }^{18}$ Bettina Konte, ${ }^{18}$ Panos Roussos, ${ }^{19,20,21}$ Stella Giakoumaki, ${ }^{22}$

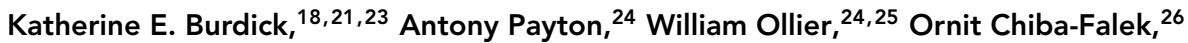 \\ Deborah K. Attix, ${ }^{26,27}$ Anna C. Need, ${ }^{28}$ Elizabeth T. Cirulli, ${ }^{29}$ Aristotle N. Voineskos, ${ }^{30}$ \\ Nikos C. Stefanis, ${ }^{31,32,33}$ Dimitrios Avramopoulos, ${ }^{34,35}$ Alex Hatzimanolis, ${ }^{31,32,33}$ Dan E. Arking, ${ }^{35}$

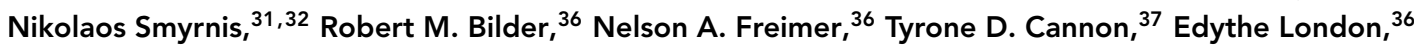 \\ Russell A. Poldrack, ${ }^{38}$ Fred W. Sabb, ${ }^{39}$ Eliza Congdon, ${ }^{36}$ Emily Drabant Conley, ${ }^{40}$ Matthew A. Scult, ${ }^{41}$ \\ Dwight Dickinson, ${ }^{42}$ Richard E. Straub, ${ }^{43}$ Gary Donohoe, ${ }^{44}$ Derek Morris, ${ }^{44}$ Aiden Corvin, ${ }^{45}$ \\ Michael Gill, ${ }^{45}$ Ahmad R. Hariri, ${ }^{41}$ Daniel R. Weinberger, ${ }^{43}$ Neil Pendleton, ${ }^{46}$ Panos Bitsios, ${ }^{47}$ \\ Dan Rujescu, ${ }^{18}$ Jari Lahti, ${ }^{11,48}$ Stephanie Le Hellard, ${ }^{6,9}$ Matthew C. Keller, ${ }^{49}$ Ole A. Andreassen, $6,7,50$ \\ David C. Glahn, ${ }^{4}$ Anil K. Malhotra, ${ }^{3,51,52}$ and Todd Lencz ${ }^{3,51,52}$ \\ ${ }^{1}$ Institute of Mental Health, Singapore \\ ${ }^{2}$ BrainWorkup, LLC, Los Angeles, CA, USA \\ ${ }^{3}$ Division of Psychiatry Research, Zucker Hillside Hospital, Glen Oaks, NY, USA \\ ${ }^{4}$ Department of Psychiatry, Yale University School of Medicine, New Haven, CT, USA \\ ${ }^{5}$ Department of Medical Genetics, Oslo University Hospital, University of Bergen, Oslo, Norway \\ ${ }^{6}$ NORMENT, K. G. Jebsen Centre for Psychosis Research, University of Bergen, Bergen, Norway \\ ${ }^{7}$ Division of Mental Health and Addiction, Oslo University Hospital, Oslo, Norway \\ ${ }^{8}$ Department of Psychology, University of Oslo, Oslo, Norway \\ ${ }^{9}$ Dr. Einar Martens Research Group for Biological Psychiatry, Center for Medical Genetics and Molecular Medicine, \\ Haukeland University Hospital, Bergen, Norway \\ ${ }^{10}$ Department of Biological and Medical Psychology, University of Bergen, Norway \\ ${ }^{11}$ Institute of Behavioural Sciences, University of Helsinki, Helsinki, Finland \\ ${ }^{12}$ Institute for Molecular Medicine Finland (FIMM), University of Helsinki, Finland \\ ${ }^{13}$ Wellcome Trust Sanger Institute, Wellcome Trust Genome Campus, Cambridge, UK \\ ${ }^{14}$ Department of Medical Genetics, University of Helsinki and University Central Hospital, Helsinki, Finland \\ ${ }^{15}$ Department of General Practice, University of Helsinki and Helsinki University Hospital, Helsinki, Finland \\ ${ }^{16}$ National Institute for Health and Welfare, Helsinki, Finland \\ ${ }^{17}$ Folkhälsan Research Center, Helsinki, Finland \\ ${ }^{18}$ Department of Psychiatry, Martin Luther University of Halle-Wittenberg, Halle, Germany \\ ${ }^{19}$ Department of Psychiatry, Icahn School of Medicine at Mount Sinai, New York, NY, USA \\ ${ }^{20}$ Department of Genetics and Genomic Science and Institute for Multiscale Biology, Icahn School of Medicine at Mount \\ Sinai, New York, NY, USA \\ ${ }^{21}$ Mental Illness Research, Education, and Clinical Center (VISN 2), James J. Peters VA Medical Center, Bronx, NY, USA \\ ${ }^{22}$ Department of Psychology, University of Crete, Rethymnon, Greece \\ ${ }^{23}$ Department of Psychiatry at Brigham and Women's Hospital, Harvard Medical School, Boston, MA, USA \\ ${ }^{24}$ Centre for Epidemiology, Division of Population Health, Health Services Research \& Primary Care, The University of \\ Manchester, Manchester, UK \\ ${ }^{25}$ Centre for Integrated Genomic Medical Research, Institute of Population Health, University of Manchester, Manchester, \\ UK \\ ${ }^{26}$ Department of Neurology, Bryan Alzheimer's Disease Research Center, and Center for Genomic and Computational \\ Biology, Duke University Medical Center, Durham, NC, USA
}

RECEIVEd 4 May 2018; ACCePted 23 May 2018. First published online 13 July 2018.

AdDress fOr CORrespondence: Todd Lencz, Zucker Hillside Hospital, Division of Psychiatry Research, 75-59 263rd Street, Glen Oaks, NY 11004, USA. E-mail: tlencz@northwell.edu 
${ }^{27}$ Psychiatry and Behavioral Sciences, Division of Medical Psychology, and Department of Neurology, Duke University Medical Center, Durham, NC, USA

${ }^{28}$ Division of Brain Sciences, Department of Medicine, Imperial College, London, UK

${ }^{29}$ Human Longevity, Inc., Durham, NC, USA

${ }^{30}$ Campbell Family Mental Health Institute, Centre for Addiction and Mental Health, University of Toronto, Toronto, Canada

${ }^{31}$ Department of Psychiatry, National and Kapodistrian University of Athens Medical School, Eginition Hospital, Athens, Greece

${ }^{32}$ University Mental Health Research Institute, Athens, Greece

${ }^{33}$ Neurobiology Research Institute, Theodor-Theohari Cozzika Foundation, Athens, Greece

${ }^{34}$ Department of Psychiatry, Johns Hopkins University School of Medicine, Baltimore, MD, USA

${ }^{35}$ McKusick-Nathans Institute of Genetic Medicine, Johns Hopkins University School of Medicine, Baltimore, MD, USA

${ }^{36}$ UCLA Semel Institute for Neuroscience and Human Behavior, Los Angeles, CA, USA

${ }^{37}$ Department of Psychology, Yale University, New Haven, CT, USA

${ }^{38}$ Department of Psychology, Stanford University, Palo Alto, CA, USA

${ }^{39}$ Robert and Beverly Lewis Center for Neuroimaging, University of Oregon, Eugene, OR, USA

${ }^{40} 23$ and Me, Inc., Mountain View, CA, USA

${ }^{41}$ Laboratory of NeuroGenetics, Department of Psychology \& Neuroscience, Duke University, Durham, NC, USA

${ }^{42}$ Clinical and Translational Neuroscience Branch, Intramural Research Program, National Institute of Mental Health, National Institutes of Health, Bethesda, MD, USA

${ }^{43}$ Lieber Institute for Brain Development, Johns Hopkins University Medical Campus, Baltimore, MD, USA

${ }^{44}$ Neuroimaging, Cognition \& Genomics (NICOG) Centre, School of Psychology and Discipline of Biochemistry, National University of Ireland, Galway, Ireland

${ }^{45}$ Neuropsychiatric Genetics Research Group, Department of Psychiatry and Trinity College Institute of Neuroscience, Trinity College Dublin, Dublin, Ireland

${ }^{46}$ Division of Neuroscience and Experimental Psychology/School of Biological Sciences, Faculty of Biology Medicine and Health, University of Manchester, Manchester Academic Health Science Centre, Salford Royal NHS Foundation Trust, Manchester, UK

${ }^{47}$ Department of Psychiatry and Behavioral Sciences, Faculty of Medicine, University of Crete, Heraklion, Greece

${ }^{48} \mathrm{Helsinki}$ Collegium for Advanced Studies, University of Helsinki, Helsinki, Finland

${ }^{49}$ Institute for Behavioral Genetics, University of Colorado, Boulder, CO, USA

${ }^{50}$ Institute of Clinical Medicine, University of Oslo, Oslo, Norway

${ }^{51}$ Department of Psychiatry, Hofstra Northwell School of Medicine, Hempstead, NY, USA

${ }^{52}$ Center for Psychiatric Neuroscience, Feinstein Institute for Medical Research, Manhasset, NY, USA

Hill (Twin Research and Human Genetics, Vol. 21, 2018, 84-88) presented a critique of our recently published paper in Cell Reports entitled 'Large-Scale Cognitive GWAS Meta-Analysis Reveals Tissue-Specific Neural Expression and Potential Nootropic Drug Targets' (Lam et al., Cell Reports, Vol. 21, 2017, 2597-2613). Specifically, Hill offered several interrelated comments suggesting potential problems with our use of a new analytic method called Multi-Trait Analysis of GWAS (MTAG) (Turley et al., Nature Genetics, Vol. 50, 2018, 229-237). In this brief article, we respond to each of these concerns. Using empirical data, we conclude that our MTAG results do not suffer from 'inflation in the FDR [false discovery rate]', as suggested by Hill (Twin Research and Human Genetics, Vol. 21, 2018, 84-88), and are not 'more relevant to the genetic contributions to education than they are to the genetic contributions to intelligence'.

Keywords: GWAS, general cognitive ability, nootropics, gene expression, neurodevelopment, synapse, calcium channel, potassium channel, cerebellum

Hill (2018) presents a critique of our recently published paper in Cell Reports entitled 'Large-Scale Cognitive GWAS Meta-Analysis Reveals Tissue-Specific Neural Expression and Potential Nootropic Drug Targets' (Lam et al., 2017). Specifically, Hill offers several interrelated comments suggesting potential problems with our use of a new analytic method called Multi-Trait Analysis of GWAS (MTAG) (Turley et al., 2018). Below, we respond to each of these concerns. For context, in our paper (Lam et al., 2017), MTAG was applied to two sets of genomewide association study (GWAS) results: One for cognitive ability $\left(\mathrm{GWAS}_{\mathrm{COG}}\right)$, and the other for educational attainment $\left(\mathrm{GWAS}_{\mathrm{EDU}}\right)$.

First, Hill (2018) suggests that our GWAS $_{\mathrm{COG}}$ and GWAS $_{\mathrm{EDU}}$ datasets 'differ a great deal in power' and that
MTAG is not appropriate when the traits examined are 'highly dissimilar' with respect to power. As noted by the creators of MTAG (Turley et al., 2018), power for a GWAS can be quantified by the non-centrality parameter (NCP) of the mean $\chi^{2}$ statistic, which scales with sample size and heritability. In our study, mean $\chi^{2}$ was 1.245 for GWAS ${ }_{\mathrm{COG}}$ and 1.638 for GWAS $_{\mathrm{EDU}}$, and the ratio of the NCPs is equal to 0.384 . Are these 'highly dissimilar' by the standards of MTAG?

The term 'highly dissimilar' is a subjective appraisal, while we seek to address the question quantitatively. Fortunately, the developers of MTAG provided simulations across a range of scenarios in order to establish benchmarks for when MTAG might be problematic; see Turley et al. 
(2018, Supplementary Figure 1.2.a). The scenarios tested included: (a) both GWAS have equally low power (mean $\chi^{2}=1.1$ for each; NCP ratio $\left.=1\right)$; (b) one low-powered GWAS (mean $\chi^{2}=1.1$ ) and one moderate-powered GWAS (mean $\chi^{2}=1.4$ ), resulting in an NCP ratio of 0.25 ; (c) one low-powered GWAS (mean $\chi^{2}=1.1$ ) and one highpowered GWAS (mean $\chi^{2}=2.0$ ), with NCP ratio $=0.1$. Of these, only the last of these scenarios resulted in problematic inflation of the false discovery rate (FDR). By contrast, our NCP ratio was most comparable to (and somewhat more favorable than) scenario (b) above, which showed virtually no increase in FDR relative to the scenario in which the two GWAS were equally powered (scenario a).

Most importantly, Hill (2018) raises the specific concern that the difference in power between GWAS $_{\mathrm{COG}}$ and

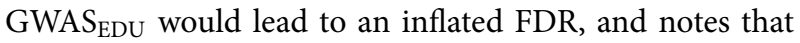
our paper (Lam et al., 2017) did not report the 'max FDR' calculation as made possible by the MTAG software. Here, we can report that the max FDR calculation for the MTAG results reported in Lam et al. (2017) was 0.0068. This is nearly an order of magnitude below the commonly accepted 0.05 standard for false discovery. Moreover, our max-FDR value is comparable to those reported by Turley et al. (2018) for their empirical MTAG study of depression, neuroticism, and subjective wellbeing. Placed in context of the specific results of Lam et al. (2017), in which 70 independent loci (with 82 independent lead SNPs) were identified by MTAG, a FDR of 0.0068 suggests that between 0 and 1 of these loci is false.

Hill (2018) points to the pattern of genetic correlations reported in our manuscript (Lam et al., 2017, Supplementary Table 14) as evidence that the polygenic signal derived from our MTAG 'is indistinguishable from that of education' This characterization is inaccurate for several reasons. First, and most simply, our Supplementary Table 14 demonstrates the set of genetic correlations for the MTAG data differ from those of educational attainment (from the Okbay et al., 2016 dataset) in many cases by an absolute value (for $r_{\mathrm{g}}$ ) of 0.10 or greater. As an important example, the polygenic signal from MTAG demonstrated a strong correlation with childhood IQ $\left(r_{\mathrm{g}}=0.86\right)$, which was virtually identical to that observed between childhood IQ and GWAS COG $\left(r_{\mathrm{g}}=0.87\right)$; by contrast, the correlation between childhood

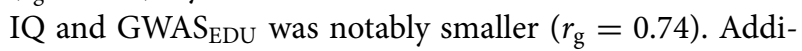
tionally, there are several instances in which the MTAG correlations are not only more similar to those for GWAS $_{\mathrm{COG}}$ than for $\mathrm{GWAS}_{\mathrm{EDU}}$, but the absolute value of the MTAG correlations are marginally greater than those observed for either $\mathrm{GWAS}_{\mathrm{COG}}$ or $\mathrm{GWAS}_{\mathrm{EDU}}$; for example, for infant head circumference, the genetic correlation $\left(r_{\mathrm{g}}\right)$ with MTAG results is $0.2962(p=1.88 \mathrm{E}-07)$, as compared to its correlation with either GWAS $_{\mathrm{COG}}(0.2705 ; p=2 \mathrm{E}-04)$ or $\mathrm{GWAS}_{\mathrm{EDU}}$ (0.2597; $p=2.71 \mathrm{E}-06)$.

While not mentioning these counterexamples to his argument, Hill (2018) focuses on the genetic correlations be- tween our MTAG results and other educational variables, which are indeed large $\left(r_{\mathrm{g}}>0.90\right)$. However, Hill (2018) elides the fact that the calculation method employed by LD score regression is known to sometimes produce values for $r_{\mathrm{g}}>1$, if the variables are so highly similar as to be self-same (Walters, 2016). Such values (between GWAS and other virtually identical educational variables) are accurately reported in our Supplementary Table 14, but are truncated to 1 in Hill's partial reproduction of our table (Hill, 2018, Table 1), thereby implying a greater similarity than may be actually present. Importantly, the genetic correlation between the MTAG results and its two constituent GWAS provide evidence contrary to Hill's claim that the 'Intelligence-MTAG phenotype derived by Lam et al. (2017) is more similar to Education than it is to Cognitive ability'. Specifically, the correlation between MTAG and GWAS COG $_{\text {. }}$ $\left(r_{\mathrm{g}}=0.96, S E=0.0058\right)$ is significantly greater than that between MTAG and GWAS $\mathrm{EDU}_{\mathrm{g}}\left(r_{\mathrm{g}}=0.91, S E=0.0052\right)$. Further, Hill (2018) calls attention to the intriguing pattern of results that obtains for schizophrenia and bipolar disorder, which we also noted with interest in our published paper (Lam et al., 2017). Rather than invalidating our approach, we believe that this finding highlights a biologically meaningful set of relationships that we have extensively analyzed in the context of a subsequent manuscript that is currently in preparation. Finally, it is important to note that the overall pattern of genetic correlations is highly similar between all three sets of measures, as is evident in Figure 5 of Lam et al. (2017).

While the foregoing paragraphs address each of the specific points raised by Hill (2018), we wish to note two additional facts reported in our paper (Lam et al., 2017) that weigh against Hill's conclusion. First, our leave-oneout analyses (Lam et al., 2017, Figure 3) demonstrate that prediction of held-out samples, phenotyped for cognitive ability, are better for MTAG than for either GWAS $_{\mathrm{COG}}$ or GWAS $_{\text {EDU }}$ alone. This finding supports our interpretation that MTAG is boosting polygenic signal for cognition, and does not support the conclusion of Hill (2018) that the MTAG polygenic signal is indistinguishable from that of education'. Second, as demonstrated in Figure 2 (and associated text) in Lam et al. (2017), the top results (genome-wide significant loci) emerging from MTAG show notable differences from those emerging from GWAS $_{\mathrm{EDU}}$, but are almost a complete superset of those emerging from GWAS $_{\mathrm{COG}}$. Furthermore, based on results we have already seen, we are confident that the novel loci we have identified will receive additional support in forthcoming, larger GWAS studies of cognitive ability that do not incorporate educational attainment data using MTAG.

For all of the empirical reasons cited above, we believe our MTAG results do not suffer from 'inflation in the FDR [false discovery rate]', as suggested by Hill (2018), and are not 'more relevant to the genetic contributions to education than they are to the genetic contributions to intelligence. We 
continue to be confident of the evidence reported in Cell Reports (Lam et al., 2017). As suggested above, further efforts underway by the COGENT consortium and other collaborators continue to build additional supporting and clarifying evidence with regard to these issues. Specifically, we have additional large-scale GWAS data, and we continue to further investigate the genetic architecture of cognition and potential downstream implications to neuropsychiatric disease and other health outcomes.

\section{Acknowledgments}

The authors gratefully acknowledge the thoughtful comments and feedback on an earlier draft of this manuscript from Patrick Turley and Raymond K. Walters (the first author and second author of the paper that developed MTAG).

\section{References}

Hill, W. D. (2018). Comment on 'large-scale cognitive GWAS meta-analysis reveals tissue-specific neural expression and potential nootropic drug targets' by Lam et al. Twin Research and Human Genetics, 21, 84-88.

Lam, M., Trampush, J. W., Yu, J., Knowles, E., Davies, G., Liewald, D. C., ... Lencz, T. (2017). Large-scale cognitive GWAS meta-Analysis reveals tissue-specific neural expression and potential nootropic drug targets. Cell Reports, 21, 2597-2613.

Okbay, A., Beauchamp, J. P., Fontana, M. A., Lee, J. J., Pers, T. H., Rietveld, C. A., ... Benjamin, D. J. (2016). Genome-wide association study identifies 74 loci associated with educational attainment. Nature, 533, 539542.

Turley, P., Walters, R. K., Maghzian, O., Okbay, A., Lee, J. J., Fontana, M. A., ... Social Science Genetic Association Consortium. (2018). Multi-trait analysis of genome-wide association summary statistics using MTAG. Nature Genetics, 50, 229-237.

Walters, R. T. (2016, April 25). Response to query on LD score regression user forum. Retrieved February 1, 2018, from https:/groups.google.com/forum/\#!topic/ldsc_users/ UY2vlI4AJbc 